\title{
Automatic diagnostic of transmission lines based on ultraviolet inspection
}

\author{
Phuong Nguyen ${ }^{1, *}$, Sergey Dudkin ${ }^{1}$, and Chenzai Kong ${ }^{2}$ \\ ${ }^{1}$ Peter the Great St. Petersburg Polytechnic University, Electrical Department, 194021 Polytechnicheskaya 29, Russia \\ ${ }^{2}$ Xi'an University of Science and Technology, Electrical department, Yanta Road 58, China
}

\begin{abstract}
Evaluation of the technical condition, reliability of the insulation of electrical equipment is an actual problem. It is confirmed by experience and statistics of operation at power plants and railway facilities. The combination of an unmanned aerial vehicle with UV-camera and software based on neural networks allows us to effectively diagnose long power lines. To increase the effectiveness of non-contact inspection of power lines, especially in hard-to-reach areas, more compact mobile solutions should be used which include an UV-camera and an unmanned aerial vehicle (UAV). The aircraft market currently has significant growth, that allows to bring the diagnostic experience to a new level by attaching an UV-camera to an aerial device, which will have a tremendous effect on examining long power lines. But we faced one problem related to the absence of any scientific basis for this method of examination, so it was decided to conduct experiments in a laboratory of St. Petersburg Polytechnic University. The results of experiments are presented in the work.
\end{abstract}

\section{Introduction}

Evaluation of the technical condition, reliability of the insulation of electrical equipment is an actual problem. It is confirmed by experience and statistics of operation at power plants and railway facilities.

Monitoring the state of external insulation of electrical equipment is one of the main types of diagnostics in the operation of electrical networks and equipment, since insulation defects lead to the ionization of air voids and the generation of partial discharges or corona. These discharge processes gradually destroy insulation, cause energy losses and radio interference, etc $[1,2]$. In the practice of operation, the normal state of insulation is the absence of partial discharges and corona, and their presence indicates possible defects or contamination of insulation [3]. The need to determine the resistance to surface partial discharges increases in the case of using new polymeric materials due to their properties $[4,5,6$, 7].

At the moment, contact (insulation resistance measurement, ultrasonic method, voltage measurement on insulators using a measuring rod) and non-contact (acoustic, thermal imaging, ultraviolet) methods are used for technical diagnostics of insulation [7, 8].

Non-contact diagnostic methods have an undoubted advantage over a contact one in the absence of necessity to disconnect a measurement object. In addition, UV and
IR methods and can at all point to a probable defect, visualizing its place on the diagnosed object [9, 10,].

Considering of the main methods of UV inspection $[11,12]:$

1. Location of the centers of the corona category on power line wires [13], etc.

2. Monitoring the state of suspension and supporting porcelain, glass and polymer insulation.

3. Assessment of the degree of contamination of insulation.

All methods are based on an estimate of the intensity of the discharge: the number of flashes, the size of the UV spot. Depending on the intensity of the outbreaks observed, the external insulation status of electrical equipment is assessed according to the 3 categories of defect development: developing, pre-emergency and emergency [14, 15].

To increase the effectiveness of non-contact inspection of power lines, especially in hard-to-reach areas, more compact mobile solutions should be used which include an UV-camera and an unmanned aerial vehicle (UAV). The aircraft market currently has significant growth, that allows to bring the diagnostic experience to a new level by attaching an UV-camera to an aerial device, we have a tremendous effect in examining long power lines.

\footnotetext{
* Phuong Nguyen: nguyenphuong95@mali.ru
} 


\section{Methods}

UV-cameras from CoroCam and Ofil are widely used in Russia. These brands produce cameras that can be used in the daytime, when solar activity is high. In the company "Rosseti" the cameras of both companies are exploited. And at the moment, the list has replenished with a new company from Saint-Petersburg for energetic branch

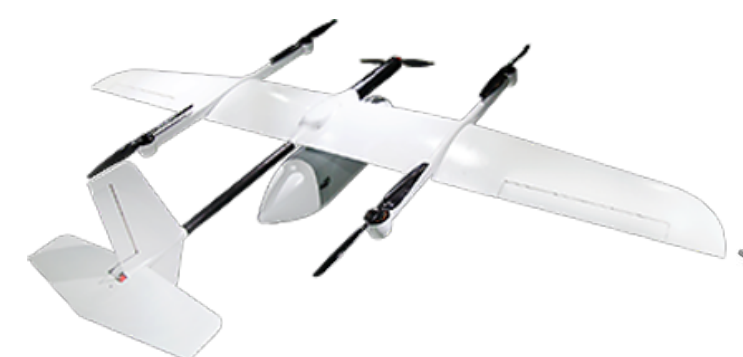

Fig.1. Petrol and electric unmanned aerial vehicle.

But we faced one problem related to the absence of any scientific basis for this method of examination, so it was decided to conduct experiments in a laboratory of St. Petersburg Polytechnic University. The experiments described in this article were conducted using the Ofil DayCor Superb camera.

\section{Results and Discussion}

We used guidelines [18] written specifically for laboratory work on a high-voltage test bench, which is located on the territory of the High Voltage Engineering Department.

\subsection{Conditions influencing the indications of the UV-camera}

The sensitivity of the camera detector is the most important characteristic determining the reliability of ultraviolet diagnostics. The UV range of the electromagnetic spectrum lies to the left of the visible range and is approximately $200-400 \mathrm{~nm}[16,17]$. The spectrogram of the corona, with an increase in scale 1000 times, is highlighted in blue. The spectrum of the corona view corresponds to the electromagnetic radiation of atmospheric nitrogen when it is ionized. The mechanism of this process, in particular, can help to eliminate the defect or contamination of insulation.

It follows from Fig. 2 that the spectrum of the corona has two pronounced maxima arising at 340 and $360 \mathrm{~nm}$. At the same time, in these ranges the strength of solar radiation is also great.

In order to be able to diagnose the insulator in daylight, in the modern electron-optical of UV-camera a range of $240-280 \mathrm{~nm}$ is used. In this range, solar radiation almost completely absorbs molecules of atmospheric ozone [3], and isolation can be observed with small hindrances or their absence.

Factors, influencing results of UV-inspection $[13,16]$ :

- wind speed;

- air humidity;

- ambient temperature; which is developing its own ultraviolet camera while has an option for examining of power lines with UAV.

Having carried out a number of analyzes of the offers of UAV available on the market, we identified two key companies that can provide a fairly effective solutions (fig.1) that can make long flights and for which it is possible to program the flight route without operator intervention.

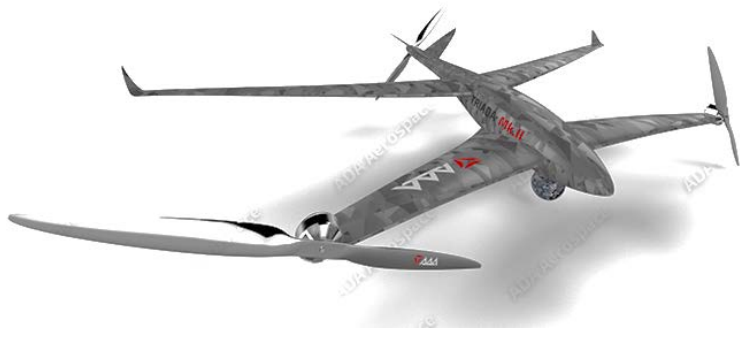

- the distance to the surveyed object;

- viewing angle;

- Presence of precipitation;

- degree of insulation contamination [17].

This method of diagnostics has not been sufficiently studied yet and needs to be refined and verified in order to obtain more reliable results. For this purpose, experiments were carried out to solve the following problems:

- Check the camera's capabilities to measure the characteristics of the crown.

- Make sure that the results obtained are correct during the diagnostics in the power system.

\subsection{Calibration}

Using the camera, we received a combined image, processed from two matrices. The gray rectangle limits the area where the number of flashes is counted. For example, we can observe the crown at the end of the needle conductor and on its bends, as well as on the copper wire. At the bottom of the photo is information about the shooting mode, the current values of the gain and the number of flashes.

Before the experiments, the diagnostic device was calibrated. From the obtained data, it was found that with an increase in the sensitivity gain of the matrix, the error in the readings of the UV camera also increases.

\subsection{Determination of the minimum sensitivity by the UV-camera}

For this experiment, an oscilloscope was used to measure the corona current and the power of the corona losses. At different magnification values: 40, 80, 120 and 200, the detection voltage of the corona of the UV camera and the oscilloscope differed for the needle conductor. Measurement of the corona discharge current allowed to fix the beginning of the corona discharge at a voltage of $35 \mathrm{kV}$, while the camera fixed the corona at a voltage of $32 \mathrm{kV}$. But the advantage of the camera over the method based on measuring the corona current was reduced to a 
minimum when the observation object was changed to a wire where the detection voltage of the corona was the same: $35 \mathrm{kV}$.

\subsection{Determine the dependence of the UV-camera readings on the survey distance}

The source of the crown was the needle guide. The dependence of the number of flashes on the shooting distance is clearly shown in Fig. 2. Due to the random character of the points on the graph, it is necessary to use power approximation to obtain a visual curve. A similar operation will be carried out for other graphs in this work, but with different approximation methods. The equation for the graph is:

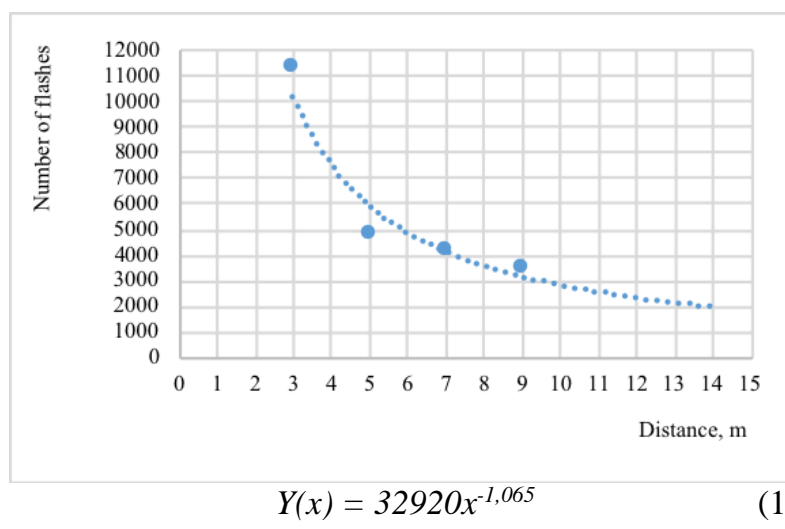

Fig. 2. Graph of the dependence of the number of flashes on the distance to the object.

The dependence of the number of flares on the distance to the object has a power-law character. The difference between the UV camera readings for distances of 3 and 5 meters is the most significant: at a distance of 3 meters the number of recorded outbreaks was 2.4 times higher than the readings at a distance of 5 meters. At a subsequent distance from the object under examination, the readings decreased slightly. In practice, the UV inspection is usually carried out at a distance of not less than 5 meters from the facility, so the impact on the conclusion about UFD will be influenced by cases of shooting from a closer distance. The similar picture we can observe in $[19,20]$.

\subsection{Comparison of the dependence of the UV- flashes and the corona power loss on the voltage for a copper wire and a needle conductor.}

\subsubsection{Needle conductor}

The first experiment was carried out for a needle conductor with a gain of 120 . For different voltage values, 21 flashes were taken every 3 seconds (the refresh rate of the camera readings). To plot the graph below, a linear approximation was used:

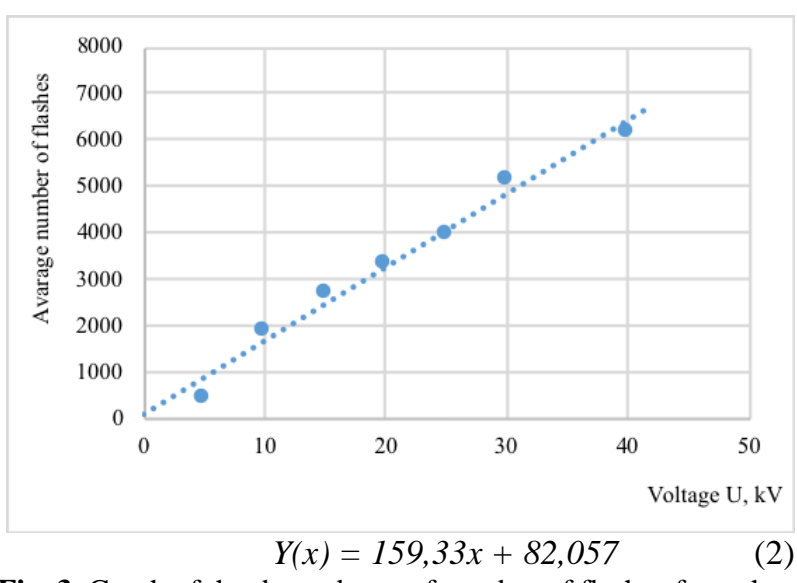

Fig. 3. Graph of the dependence of number of flashes from the voltage for the needle conductor at a gain of 120 .

The graph in Fig. 3 is linear. The voltage at which the corona discharges on the wire affected the characteristic of the corona from the needle conductor came at a voltage of $45 \mathrm{kV}$, so the experiment was not conducted with a high voltage.

\subsubsection{Copper wire}

For this experiment, a copper wire with a cross section of $2 \mathrm{~mm}$ was used. Equation for the graphs 1, 2, and 3 with polynomial approximation:

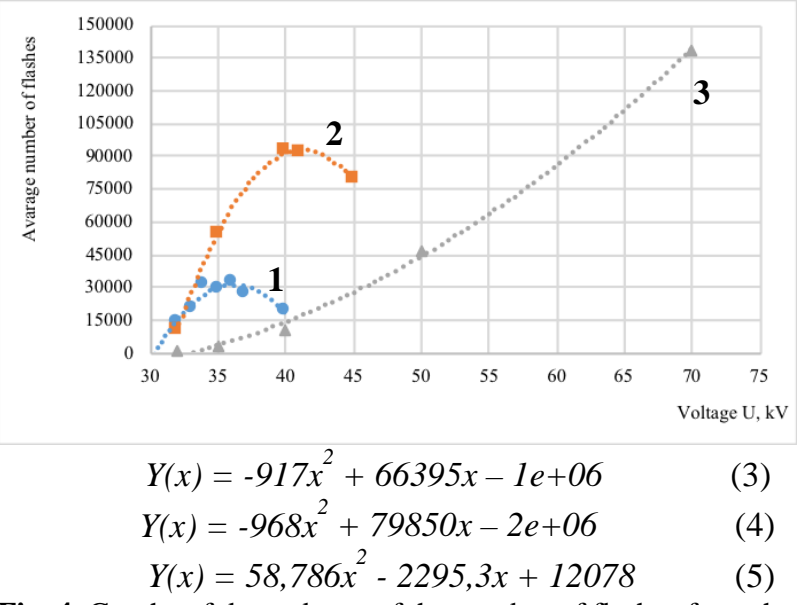

Fig. 4. Graphs of dependence of the number of flashes from the voltage for a copper wire at different gain factors: 1) 120, 2) 80, 3) 40.

Analyzing the obtained graphs, we see that for the amplification factors 120 and 80, when a certain voltage is reached, an inflection appears, after which the number of flashes decreases. This phenomenon can be explained by the high sensitivity of the camera, which affects its selectivity: flashes on the matrix merge, and accordingly, the calculation of their number becomes incorrect. When the gain was reduced to 40 , the kink was not observed, but presumably it should occur at a high voltage, the achievement of which was not possible due to the limitations of the test bench.

It is also worth noting that with no gain the result will not be absolutely accurate, since at high values of sensitivity, flashes can merge, and at small values, it cannot be detected. 
An oscilloscope was used to determine the power of corona losses. The power of losses is determined by the formula:

$$
P(S)=S K_{u} K_{q} f \text {, }
$$

where $S$ is the area limited by the curve of the voltcoulomb characteristics, $\operatorname{div}^{2}$.; $K_{u}$ and $K_{q}$ - scale factors for voltage and charge, respectively; $f$ - a frequency equal to $50 \mathrm{~Hz}$.

Scale factors:

$$
K_{u}=\frac{U}{h_{y}}
$$

where $U$ is the voltage value on the wire, $\mathrm{V} ; h_{y}$ - the maximum deviation of the beam from the zero line vertically, div.

$$
K_{q}=h_{x} C 3 \text {, }
$$

where $h_{x}$ is the horizontal sensitivity of the oscilloscope, equal to $0.2 \mathrm{~V} / \mathrm{div}$; $\mathrm{C} 3=0.47 \cdot 10^{-6} \mathrm{~F}$.

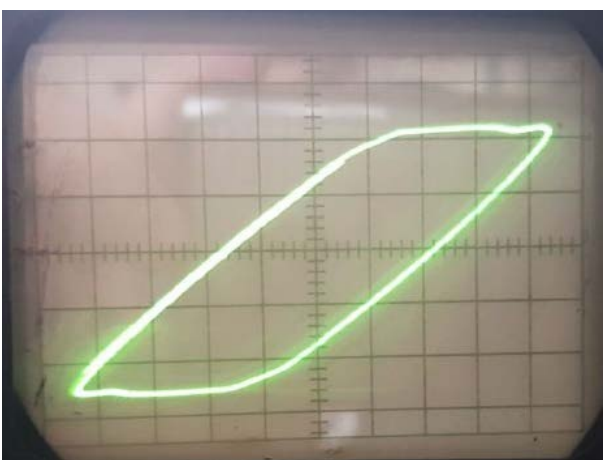

Fig. 5. An example of measuring power loss on corona.

For a graph of the dependence of the power loss per crown on voltage, a linear approximation of the equation of which is presented below:

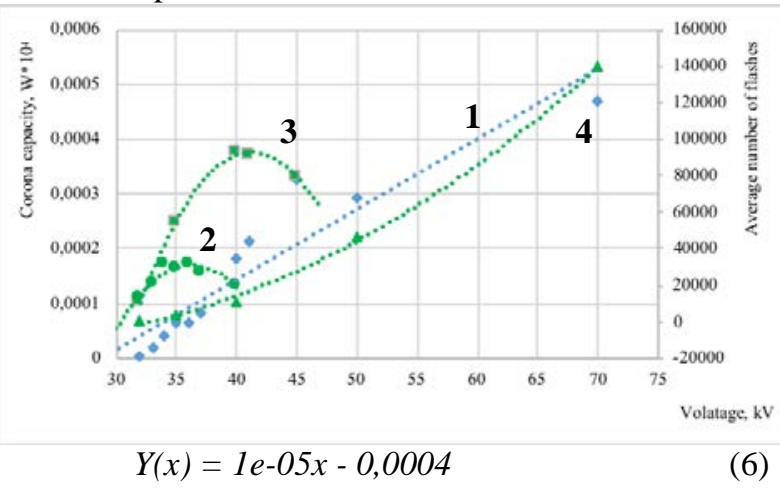

Fig. 6. Graphs of dependence cf. number of flares and power loss per crown (1) of the voltage for a copper wire at different gain factors: 2) 120, 3) 80, 4) 40 .

According to the graph in fig. 6, the dependence of the power of losses on the corona on the voltage is linear, which corresponds to the theoretical curve.

\section{Conclusions}

According to the results of the experiments, it can be said that UV-inspection is a fairly good method for the rapid diagnosis of electrical equipment [20, 21]. But this method has its advantages and disadvantages, which must be taken into account when conducting the survey:
1. The UV-camera has a high sensitivity, comparable with the sensitivity of the laboratory installation.

2. The distance to the subject has a strong influence on the UV-camera, but with its increase, the number of flashes varies slightly. This fact allows you to conduct a survey from a certain distance without a strong scatter in the camera.

3. The camera has a limit of measuring the number of flashes per unit of time due to the resolution of the sensor, which requires a reduction in the gain, but at the same time, weaker signals are lost.

4 . The curves of the dependence of the power of losses on the corona and the number of flashes on the voltage have a similar tendency, which makes it possible to bring the camera to the traditional physical quantity used, the power of the corona loss.

5. It is necessary to take into account the errors and peculiarities of the camera, which do not allow unambiguous interpretation of the result of the survey, as described in the instructions. Therefore, it is necessary to develop a methodology that takes into account these features.

We got the first results of laboratory measurements that gave us the opportunity to associate a real physical quantity with the readings of the camera. At the moment, collected data of UV-inspection includes 280,000 photos from actual exploitation, but due to the absence of a clear understanding of the nature of the defect in the presented photos, additional experiments are necessary to be provided. Future experiments in the climatic chamber will soon be carried out. That fact will allow us to increase the amount of data for the correct training of neural networks, which will become the basis for an automatic system to diagnostic of power transmission lines [22].

The combination of an unmanned aerial vehicle with UV-camera and software based on neural networks allows us to effectively diagnose long power lines. And this is the future we are striving for.

\section{References}

1.R.S. Arbuzov, Modern methods of diagnostics of overhead power lines. Novosibirsk: Science, 136 (2009)

2.Field Guide: Daytime Discharge Inspection of Transmission and Distribution Overhead Lines and Substations Electrical power research institute (2013)

3.G.N. Mohamad Partial Discharge Signatures of Defects in Insulation Systems Consisting of Oil and Oilimpregnated Paper Licentiate Thesis Stockholm, Sweden (2012)

4. Bin Ma. Effects of corona and ozone exposure on properties of polymeric materials for high voltage outdoor applications / Sweden 125 (2011)

5.L. D. Ginzburg, Solid insulation of high-voltage structures for indoor installations Energoatomizdat. St. Petersburg (1992)

6. CIGRE Study Committee 22, "Insulators - review of in-service diagnostic testing of composite insulators", (2014) 
7.R.S. Arbuzov, A.G. Ovsyannikov. Assessment of the degree of contamination of the insulation of power lines using Filin-6 / Journal PL 229-232 (2018)

8.FF Bologna, "Infrared and ultraviolet imaging techniques applied to the inspection of outdoor transmission voltage insulators"

9.W.L. Vosloo, R. Stolper, "Daylight corona discharge observation and recording system”, ISH (1997)

10. P.M. Svi. Methods and tools for diagnosing high voltage equipment M.: Energoatomizdat, 240 (1992)

11. Scientific-technical and educational journal "Electromechanics", 1, 208 (2013)

12. Ch. Lan, L. Lin, T. Mimi, B. Xingming, W. Liming, G. Zhicheng, The Ultraviolet Detection of Corona Discharge in Power Transmission Lines Energy and Power Engineering (2013)

13. Production instruction of Lenenergo PJSC on the procedure for conducting ultraviolet examination of insulation of high-voltage lines and power lines. Revision 1 (2015)

14. Transmission line reference book $345 \mathrm{kV}$ and above / General electric company Published by Electric Power Research Institute, (New York, 1980)

15. P. S. Maruvada, "Corona Performance of High-voltage Transmission Lines," New York, USA: Research Studies Press Ltd (2015)

16. Methodical instructions for remote optical monitoring of insulation of overhead power lines and AC distribution devices with voltage of $35-1150 \mathrm{kV}$. STO 56947007-29.240.003-2008 (2008)

17. A.G. Ovsyannikov, D.A. Brown, R.S. Arbuzov, V.M. Tolchin, The degree of contamination of the surface of the insulation. Evaluation of the spectrum of optical radiation discharges / / News Electrical 5(101), pp. 60-62 (2016)

18. Yu.N. Bocharov, High voltage technique. High-voltage tests and measurements: textbook (lab. Workshop) SPb.: Polytechnic Publishing House. University, 210 (2013)

19. C. Lan, L. Lin, T. Mimi, B. Xingming, W. Liming, G. Zhicheng, The Ultraviolet Detection of Corona Discharge in Power Transmission Lines. Energy and power engineering 5 (2013)

20. Y. Kim and K. Shong, "The Characteristics of UV Strength According to Corona Discharge from Polymer Insulators Using a UV Sensor and Optic Lens," IEEE Transactions on Power Delivery, 26, 3 (2011)

21. M. Lindner, S. Elstein, P. Lindner, J. M. Topaz and A. J. Phillips, "Daylight Corona Discharge Imager," Proceed-ings of 11th International Symposium on High Voltage Engineering, London, UK, (2010)

22. L. Ying, Q. Jiafeng, Zh. Jiabin, L. Chengqi, Zh. Zhenjun, Zh. Mei, Learning based Discharge Information Extraction for UltravioletImage of Electrical Equipment. Journal of Physics Conference, (2019) 